\title{
The Asymptotic Analysis of the Solution of an Elasticity Theory Problem for a Transversely Isotropic Hollow Cylinder with Mixed Boundary Conditions on the Side Surface
}

\author{
Magomed Farman Mekhtiyev', Nina Ilyinichna Fomina ${ }^{2}$, Nazaket Boyukaga Mammadova ${ }^{2}$ \\ ${ }^{1}$ Department of Applied Mathematics, Institute of Mathematics and Mechanics of National Academy of Sciences of Azerbaijan, Baku, \\ Azerbaijan \\ ${ }^{2}$ Faculty of Applied Mathematics and Cybernetics, Baku State University, Baku, Azerbaijan
}

Email address:

Fomina1109@mail.ru (N. I. Fomina)

\section{To cite this article:}

Magomed Farman Mekhtiyev, Nina Ilyinichna Fomina, Nazaket Boyukaga Mammadova. The Asymptotic Analysis of the Solution of an Elasticity Theory Problem for a Transversely Isotropic Hollow Cylinder with Mixed Boundary Conditions on the Side Surface. Science Journal of Applied Mathematics and Statistics. Vol. 4, No. 6, 2016, pp. 269-275. doi: 10.11648/j.sjams.20160406.14

Received: September 23, 2016; Accepted: October 7, 2016; Published: November 3, 2016

\begin{abstract}
The problem of elasticity theory for the transversely isotropic hollow cylinder with mixed conditions on the side surface is considered in the paper. Transcendental equations are obtained regarding the eigenvalues of the problem. The roots of the characteristic equations are studied thoroughly. The study of the eigenvalues allowed to establish the essential characteristics of the stress-strain state of an anisotropic shell in comparison with isotropic shells. Homogeneous solutions were built here.
\end{abstract}

Keywords: Theory of Elasticity, Transversely Isotropic Hollow Cylinder, Side Surface, Mixed Boundary Conditions, Stress-Strain State, Eigenvalues, Transcendental Equation, Anisotropic Shell

\section{Introduction}

The modern theory of shells is deeply developed section of the mechanics of a deformable solid. However, the calculation of shells on the basis of three-dimensional equations of the theory of elasticity is associated with considerable mathematical difficulties. Therefore, it is necessary to apply to a variety of approximate methods to simplify the calculation of shells. Many methods of bringing the three-dimensional problem to a two-dimensional one use small shell thickness compared to its other dimensions in the constructions. Among them a special place is occupied by the asymptotic method. The asymptotic methods of integrating the equations of two-dimensional shell theory obtained the great development in A. L. Goldenveiser's papers [1], [2]. V. V. Novozhilov's [3] combination of complex transformation of equations of the shell theory with the asymptotic methods is presented in K. F. Chernykh's works [4], [5]. With regard to the study of three-dimensional stress-strain state of elastic bodies the development of an asymptotic method belongs to K. Fridrix, L. Dressler [6], [7], A.L. Goldenweiser, I.I.Vorovich [8], [9], [10]. Further development of the asymptotic method went in two directions. In the first one the solution of the elasticity problem for thin bodies is carried out by means of direct integration of elasticity equations with the help of two iterative processes. This direction is developed in the works of A.L. Goldenveiser, M.I. HuseynZade, A.V. Kolos [11], [12] and L.A. Agalovyan [13].

The second approach is based on the investigation of a system of uniform solutions. The above-mentioned direction was developed by I.I. Vorovich [8], [9], D.C. Aksentyan [14], [15], O.S. Malkina [16], [17], N.N. Bazarenko [18], [19], T.A. Vilenskaya [20], Y.A. Ustinov [21], [22], [23] and M.F. Mekhtiev [24], [25].

Thus, the asymptotic method developed by A. L. Goldenweiser, I. I. Vorovich, V. B. Lidski [26] and their followers [27], [28], [29], [13], [30], [31], has made significant contribution to the development of the theory of 
plates and shells.

\section{Statement of the Problem and Its Solution}

Let the cylinder occupies a volume

$$
\Gamma=\left\{r \in\left[R_{1}, R_{2}\right], \varphi \in[0,2 \pi], z \in[-l, l]\right\}
$$

The equilibrium equations in displacements are in the form [32]:

$$
\begin{aligned}
& b_{11}\left(\Delta_{0} u_{\rho}-\frac{u_{\rho}}{\rho}\right)+\frac{\partial^{2} u_{\rho}}{\partial \xi^{2}}+\left(1+b_{13}\right) \frac{\partial^{2} u_{\xi}}{\partial \rho \partial \xi}=0 \\
& \left(1+b_{13}\right) \frac{\partial}{\partial \xi}\left(\frac{\partial u_{\rho}}{\partial \rho}+\frac{u_{\rho}}{\rho}\right)+\Delta_{0} u_{\xi}+b_{33} \frac{\partial^{2} u_{\xi}}{\partial \xi^{2}}=0
\end{aligned}
$$

here

$$
\rho=R_{0}^{-1} \cdot r, \quad \xi=R_{0}^{-1} \cdot z, \quad u_{\rho}=R_{0}^{-1} \cdot u_{r}, \quad u_{\xi}=R_{0}^{-1} \cdot u_{z}
$$

$R_{0}=1 / 2\left(R_{1}+R_{2}\right)$ is the radius of the middle surface of the shell,

$$
\begin{aligned}
& m b_{11}=2 G_{0}\left(1-v_{1} v_{2}\right), m b_{13}=2 G_{0} v_{1}(1+v), \\
& m b_{33}=2 G_{0}\left(1-v^{2}\right) \\
b_{12}= & b_{11}-2 G_{0}, E_{0}=E_{1} \cdot E^{-1}, G_{0}=G \cdot G_{1}^{-1}, \\
v_{2}= & E_{0} \cdot v_{1}
\end{aligned}
$$

$m=1-v-2 v_{1} \nu_{2}$ are dimensionless quantities, $E, G, v$ are isotropic material constants, $E_{1}, G_{1}, v_{1}$ are material constant in a plane perpendicular to the plane of isotropy.

The relations of the generalized Hooke's law are [33]:

$$
\begin{aligned}
& \sigma_{r}=G_{1}\left(b_{11} \varepsilon_{r}+b_{12} \varepsilon_{\varphi}+b_{13} \varepsilon_{z}\right) \\
& \sigma_{\varphi}=G_{1}\left(b_{12} \varepsilon_{r}+b_{11} \varepsilon_{\varphi}+b_{13} \varepsilon_{z}\right), \\
& \sigma_{z}=G_{1}\left[b_{12}\left(\varepsilon_{r}+\varepsilon_{\varphi}\right)+b_{33} \varepsilon_{z}\right], \\
& \tau_{r z}=G \varepsilon_{r z}
\end{aligned}
$$

where

$$
\begin{aligned}
\varepsilon_{r} & =\frac{\partial u_{\rho}}{\partial \rho}, \quad \varepsilon_{\varphi}=\frac{u_{\rho}}{\rho}, \quad \varepsilon_{z}=\frac{\partial u_{\xi}}{\partial \xi} \\
\varepsilon_{r z} & =\frac{\partial u_{\rho}}{\partial \xi}+\frac{\partial u_{\xi}}{\partial \rho}
\end{aligned}
$$

Suppose that the boundary conditions are given at the cylinder side surface

$$
u_{r}=0, \quad \tau_{r z}=0 \text { for } r=r_{s}(s=1,2)
$$

The nature of the boundary conditions at the ends of the cylinder is not defined yet, but we assume them so that the cylinder is in equilibrium state.
The solution (1), (3) will be sought in the form of:

$$
u_{\rho}=u(\rho) \frac{d m}{d \xi}, \quad u_{\xi}=W(\rho) m(\xi)
$$

where the function $m(\xi)$ is subjected to the condition:

$$
\frac{d^{2} m}{d \xi^{2}}-\mu^{2} m(\xi)=0 .
$$

Substituting (4) into (1) to (5), we obtain the following boundary value problem

$$
\begin{aligned}
& b_{11}\left(u^{\prime}+\frac{u}{\rho}\right)^{\prime}+\mu^{2} u+\left(1+b_{13}\right) W^{\prime}=0 \\
& \left(1+b_{13}\right) \mu^{2}\left(u^{\prime}+\frac{u}{\rho}\right)+W^{\prime \prime}+\frac{1}{\rho} W^{\prime}+b_{33} \mu^{2} W=0 \\
& u=0, \quad \mu^{2} u+W^{\prime}=0 \text { for } \rho=\rho_{s}
\end{aligned}
$$

The general solution of (6) has the form of:

$$
\begin{aligned}
& u(\rho)=\left(b_{33} \mu^{2}-\alpha_{1}^{2}\right) Z_{1}\left(\alpha_{1} \rho\right)+\left(b_{33} \mu^{2}-\alpha_{2}^{2}\right) Z_{1}\left(\alpha_{2} \rho\right), \\
& W(\rho)=-\left(b_{13}+1\right) \mu^{2}\left[\alpha_{1} Z_{0}\left(\alpha_{1} \rho\right)+\alpha_{2} Z_{0}\left(\alpha_{2} \rho\right)\right] .
\end{aligned}
$$

Here $Z_{k}(\rho)=C_{1} J_{k}(\rho)+C_{2} Y_{k}(\rho) \quad, \quad$ the functions $J_{k}(\rho), Y_{k}(\rho)$ are linearly independent solutions of the Bessel equation, $C_{1}, C_{2}$ are the arbitrary constants.

$$
\alpha_{n}=\sqrt{t_{n}}, t_{n} \text { are the roots of a quadratic }
$$

equation:

$$
\begin{aligned}
& t^{2}-2 q_{1} \mu^{2} t+q_{2} \mu^{4}=0 \\
& q_{1}=\frac{v_{1}}{v_{2}}\left(1-v_{1} v_{2}\right)^{-1}(1+v)\left(G_{o}-v_{2}\right) \\
& q_{2}=\frac{v_{1}}{v_{2}}\left(1-v_{1} v_{2}\right)^{-1}\left(1-v^{2}\right), \\
& \alpha_{n}=\mu s_{n}, s_{n}=\left[q_{1}+(-1)^{n} \sqrt{q_{1}^{2}-q_{2}}\right]^{1 / 2}
\end{aligned}
$$

By satisfying the homogeneous boundary conditions (7), we obtain the characteristic equation

$$
\begin{aligned}
& \Delta\left(\mu, \rho_{1}, \rho_{2}\right)=b_{33} \mu^{4}\left(s_{2}^{1}-s_{1}^{2}\right)^{2} \times \\
& L_{11}\left(\alpha_{1} \rho_{1}, \alpha_{1} \rho_{2}\right) L_{11}\left(\alpha_{2} \rho_{1}, \alpha_{2} \rho_{2}\right)=0
\end{aligned}
$$

where

$$
L_{11}(\alpha x, \alpha y)=J_{1}(\alpha x) \cdot Y_{1}(\alpha y)-J_{1}(\alpha y) \cdot Y_{1}(\alpha x) .
$$

The transcendental equation (10) defines a countable set of roots $\mu_{k}$, and the corresponding constants $c_{1 n}, c_{2 n}, c_{3 n}, c_{4 n}$ are proportional to the cofactors of some row of the determinant of the system. Choosing the cofactors of the elements of the 
first row as a system of solutions, the solutions to system (1) can be written as:

$$
\begin{aligned}
& u_{\rho}=\sum_{n=1}^{\infty} C_{n} U_{n}(\rho) \frac{d m_{n}}{d \xi} \\
& u_{\xi}=\sum_{n=1}^{\infty} C_{n} W_{n}(\rho) m_{n}(\xi)
\end{aligned}
$$

where $C_{n}$ are arbitrary constants.

$$
\begin{aligned}
& U_{n}(\rho)=\left[\alpha_{1}^{4}\left(b_{33} \mu_{n}^{2}-\alpha_{2}^{2}\right)^{2}-\right. \\
& \left.{ }_{-} \alpha_{1}^{2} \alpha_{2}^{2}\left(b_{33} \mu_{n}-\alpha_{1}^{2}\right)\left(b_{33} \mu_{n}-\alpha_{2}^{2}\right)\right] \times \\
& \times L_{11}\left(\alpha_{2} \rho, \alpha_{2} \rho_{2}\right) L_{11}\left(\alpha_{1} \rho_{1}, \alpha_{1} \rho_{2}\right)+ \\
& +\left[\alpha_{2}^{4}\left(b_{33} \mu_{n}^{2}-\alpha_{1}^{2}\right)^{2}-\right. \\
& \left.-\alpha_{1}^{2} \alpha_{2}^{2}\left(b_{33} \mu_{n}-\alpha_{1}^{2}\right)\left(b_{33} \mu_{n}-\alpha_{2}^{2}\right)\right] \times \\
& \times L_{11}\left(\alpha_{1} \rho, \alpha_{1} \rho_{2}\right) L_{11}\left(\alpha_{2} \rho_{1}, \alpha_{2} \rho_{2}\right) ;
\end{aligned}
$$

$$
\begin{aligned}
& W_{n}(\rho)=-\left(b_{13}+1\right)\left\{\alpha _ { 1 } \left[\alpha_{2}^{4}\left(b_{33} \mu_{n}-\alpha_{1}^{2}\right)-\right.\right. \\
& \left.{ }_{-} \alpha_{1}^{2} \alpha_{2}^{2}\left(b_{33} \mu_{n}-\alpha_{2}^{2}\right)\right] \times \\
& \times L_{01}\left(\alpha_{1} \rho, \alpha_{1} \rho_{2}\right) L_{11}\left(\alpha_{2} \rho_{1}, \alpha_{2} \rho_{2}\right)+ \\
& +\alpha_{2}\left[\alpha_{1}^{4}\left(b_{33} \mu_{n}-\alpha_{2}^{2}\right)-\right. \\
& \left.\left.{ }_{-} \alpha_{1}^{2} \alpha_{2}^{2}\left(b_{33} \mu_{n}-\alpha_{1}^{2}\right)\right] L_{01}\left(\alpha_{2} \rho, \alpha_{2} \rho_{2}\right) L_{11}\left(\alpha_{1} \rho_{1}, \alpha_{1} \rho_{2}\right)\right\}
\end{aligned}
$$

As for the stresses, they can be determined by the generalized Hooke's law [33], [34].

\section{The Asymptotic Analysis of the Problem}

The left side of equation (1.10) as an entire function of the parameter $\mu$ has a countable set of zeros with the accumulation point at infinity. For effective study of its zeros we'll assume that the shell is thin-walled.

Let us assume that

$$
\begin{aligned}
& \rho_{1}=1-\varepsilon, \rho_{2}=1+\varepsilon, \\
& \varepsilon=\left(2 R_{0}\right)^{-1}\left(R_{2}-R_{1}\right), \\
& \rho=1+\varepsilon \eta,-1 \leq \eta \leq 1
\end{aligned}
$$

We believe that $\varepsilon$ is a small parameter $\mu=0$. Substituting (12) into (10) we obtain

$$
D(\mu, \varepsilon)=\Delta\left(\mu, \rho_{1}, \rho_{2}\right)=0
$$

Equation (13) has one restricted root. From (11) we find that this corresponds to the root of the following decision:

$$
u_{\rho}=0 ; u_{\xi}=m\left[G_{0}(1+v)\right]^{-1} C_{0} \xi
$$

$$
\begin{gathered}
\sigma_{\phi}=\sigma_{r}=G_{1} v_{1} C_{0} ; \sigma_{z}=(1-v) G_{1} C_{0} \\
\tau_{r z}=0
\end{gathered}
$$

$C_{0}$ is an arbitrary constant.

Stress state corresponding to zero $\mu=0$ is equivalent to the principal vector $P$ of stresses directed along the axis of the cylinder.

$$
P=\pi(1-v) G_{1} C_{0}\left(R_{2}^{2}-R_{1}^{2}\right)
$$

Hence

$$
C_{0}=P\left[\pi(1-v) G_{1}\left(R_{2}^{2}-R_{1}^{2}\right)\right]^{-1}
$$

Let us prove that the characteristic equation (13) at $\varepsilon \rightarrow 0$ does not have any other restricted roots. For this purpose, we expand $D(\mu, \varepsilon)$ in a series in $\varepsilon$ and confine ourselves to the first terms of the expansion. We get

$$
D(\mu, \varepsilon)=16 \mu^{4}\left(s_{2}^{2}-s_{1}^{2}\right)^{2} \cdot \pi^{-2} \cdot \varepsilon^{2}[1+O(\varepsilon)]
$$

This shows that the characteristic equation has no other restricted roots besides $\mu=0$. Thus, all the roots of the characteristic equation tend to infinity as $\varepsilon \rightarrow 0$.

In principle, there could be the following limiting cases:

1) $\varepsilon \mu_{k} \rightarrow 0$ at $\varepsilon \rightarrow 0$;2) $\varepsilon \mu_{k} \rightarrow \infty$ at $\varepsilon \rightarrow 0$;

3) $\varepsilon \mu_{k} \rightarrow$ const at $\varepsilon \rightarrow 0$.

As in [32], we can prove that the cases 1 and 2 are not feasible. In the third case, we seek $\mu_{n}$ in the form:

$$
\mu_{n}=\varepsilon^{-1} \cdot \delta_{n}+0(\varepsilon) \quad(n=1,2, \ldots)
$$

As in the [32], the following cases are possible here:

1) $\mu_{1,2}= \pm s_{1} \delta_{n}, \mu_{3,4}= \pm s_{2} \delta_{n}$,

$$
\begin{gathered}
q_{1}>0, \quad q_{1}^{2}-q_{2}>0, \quad s_{1}^{2}=\delta_{n}^{2} \tau_{i} \quad(i=1,2) \\
s_{1,2}=\sqrt{q_{1} \pm \sqrt{q_{1}^{2}-q_{2}}}, \quad q_{1}^{2}>q_{2} \\
s_{1,2}=\chi+i \beta=\sqrt{q_{1} \pm i \sqrt{q_{2}-q_{1}^{2}}}, \quad q_{1}^{2}<q_{2} .
\end{gathered}
$$

2) The roots of the characteristic equation (9) are multiple.

$$
\begin{aligned}
& \mu_{1,2}=\mu_{3,4}= \pm \delta_{n} \cdot p, \\
& q_{1}>0 \quad q_{1}^{2}-q_{2}=0, \quad p=\sqrt{q_{1}}
\end{aligned}
$$

3) $\mu_{1,2}= \pm i s_{1} \delta_{n}, \quad \mu_{3,4}= \pm i s_{2} \delta_{n}$,

$$
q_{1}<0, \quad q_{1}^{2}-q_{2} \neq 0
$$

$$
\begin{array}{cc}
s_{1,2}=\sqrt{\left|q_{1}\right|+\sqrt{q_{1}^{2}-q_{2}}}, \quad & q_{1}^{2}>q_{2} \\
s_{1,2}=\sqrt{\left|q_{1}\right| \pm i \sqrt{q_{2}-q_{1}^{2}}}, & q_{1}^{2}<q_{2}
\end{array}
$$


4) $\mu_{1,2}=\mu_{3,4}= \pm i \delta_{n} p$,

$$
q_{1}<0, \quad q_{1}^{2}-q_{2}=0, \quad p=\sqrt{\left|q_{1}\right|}
$$

In cases 1 and 2, after substituting (17) into (9) and its transformation using a series expansion in $\varepsilon$ we get:

$$
\begin{gathered}
\cos \left(s_{2}+s_{1}\right) \delta_{n} \pm \cos \left(s_{2}-s_{1}\right) \delta_{n}=0 \\
\cos 2 p \delta_{n} \pm 1=0 \\
\operatorname{ch} 2 x \delta_{n} \pm \cos 2 \beta \delta_{n}=0 .
\end{gathered}
$$

With regard to the cases 3 and 4 , their results are obtained from cases 1 and 2 by a formal replacement of $s_{1}, s_{2}$ into $i s_{1}, i s_{2}$, and of $p$ into $i p$. These equations coincide with the equations determining the performance of Saint-Venant's edge effects in an anisotropic elasticity theory for a layer.

The table 1 shows the values of the coefficients for some materials:

Table 1. The coefficients for some materials.

\begin{tabular}{llll}
\hline & magnesium & cadmium & zink \\
\hline$q_{1}$ & 1,276 & 0,725 & 0,281 \\
$q_{2}$ & 1,032 & 0,425 & 0,378 \\
$q_{1}^{2}-q_{2}$ & 0,595 & 0,101 & $-0,299$ \\
\hline
\end{tabular}

\section{Asimptotic Analysis of Stress-Strain State}

We now present the first terms of the asymptotic expansions of solutions, co-responding to different groups of roots. For displacements and stresses, in the first approximation, we get two classes of solutions, the first of which corresponds to the zeros

$$
\begin{gathered}
\cos \left(s_{2}+s_{1}\right) \delta_{n}+\cos \left(s_{2}-s_{1}\right) \delta_{n}, \quad \cos 2 p \delta_{n}+1, \\
\operatorname{ch} 2 x \delta_{n}+\cos 2 \beta \delta_{n},
\end{gathered}
$$

and the second - to the zeros of the function $\cos \left(s_{2}-s_{1}\right) \delta_{n}-\cos \left(s_{2}-s_{1}\right) \delta_{n}, \quad \cos 2 p \delta_{n}-1$,

$\operatorname{ch} 2 x \delta_{n}-\cos 2 \beta \delta_{n}$

respectively, we have

$$
\begin{aligned}
& u_{\rho 0}=\varepsilon \sum_{n=1,3, \ldots}^{\infty} C_{n}\left[s_{2}^{2}\left(b_{33}-s_{1}^{2}\right) \cos s_{2} \delta_{n} \cdot \cos s_{1} \delta_{n} \eta-\right. \\
& \left.s_{1}^{2} \cos s_{1} \delta_{n} \cdot \cos s_{2} \delta_{n} \eta+0(\varepsilon)\right] \frac{d m_{n}}{d \xi} \\
& u_{\xi 0}=\left(b_{13}+1\right) s_{1} s_{2} \sum_{n=1,3, \ldots}^{\infty} C_{n} \delta_{n}\left[s_{2} \cos s_{2} \delta_{n} \cdot \sin s_{1} \delta_{n} \eta-\right. \\
& \left.s_{1} \cos s_{1} \delta_{n} \cdot \sin s_{2} \delta_{n} \eta+0(\varepsilon)\right] m_{n}(\xi) ;
\end{aligned}
$$

$$
\begin{aligned}
& \sigma_{r 0}=G_{1} s_{1} s_{2} \times \\
& \sum_{n=1,3, \ldots}^{\infty} C_{n} \delta_{n}\left[s_{2}\left(b_{13}^{2}+b_{13}-b_{11} b_{33}+b_{11} s_{1}^{2}\right) \cos s_{2} \delta_{n} \cdot \sin s_{1} \delta_{n} \eta-\right. \\
& \left.-s_{1}\left(b_{13}^{2}+b_{13}-b_{11} b_{33}+b_{11} s_{2}^{2}\right) \cos s_{1} \delta_{n} \cdot \sin s_{2} \delta_{n} \eta+0(\varepsilon)\right] \times \\
& \times \frac{d m_{n}}{d \xi} ; \\
& \sigma_{\phi 0}=G_{1} s_{1} s_{2} \times \\
& \sum_{n=1,3, \ldots}^{\infty} C_{n} \delta_{n}\left[s_{2}\left(b_{13}^{2}+b_{13}-b_{12} b_{33}+b_{12} s_{1}^{2}\right) \cos s_{2} \delta_{n} \cdot \sin s_{1} \delta_{n} \eta-\right. \\
& \left.\quad-s_{1}\left(b_{13}^{2}+b_{13}-b_{12} b_{33}+b_{12} s_{2}^{2}\right) \cos s_{1} \delta_{n} \cdot \sin s_{2} \delta_{n} \eta+0(\varepsilon)\right] \times \\
& \times \frac{d m_{n}}{d \xi} ; \\
& \sigma_{z 0}=G_{1} s_{1} s_{2} \times \\
& \sum_{n=1,3, \ldots}^{\infty} C_{n} \delta_{n}\left[s_{2}\left(b_{13} b_{33}+b_{33}-b_{12} b_{33}+b_{12} s_{1}^{2}\right) \cos s_{2} \delta_{n} \cdot \sin s_{1} \delta_{n} \eta-\right. \\
& \left.-s_{1}\left(b_{13} b_{33}+b_{33}-b_{12} b_{33}+b_{12} s_{2}^{2}\right) \cos s_{1} \delta_{n} \cdot \sin s_{1} \delta_{n} \eta+0(\varepsilon)\right] \times \\
& \times \frac{d m_{n}}{d \xi} ; \\
& \left.\quad-s_{1}^{2}\left(b_{13} s_{2}^{2}+b_{33}\right) \cos s_{1} \delta_{n} \cdot \cos s_{2} \delta_{n} \eta+0(\varepsilon)\right] m_{n}(\xi) \\
& \tau_{r z 0}=G \cdot \frac{1}{\varepsilon} \sum_{n=1,3}^{\infty} C_{n} \delta_{n}^{2}\left[s_{2}^{2}\left(b_{13} s_{1}^{2}+b_{33}\right) \cos s_{2} \delta_{n} \cdot \cos s_{1} \delta_{n} \eta-\right.
\end{aligned}
$$$$
u_{\rho 0}=\varepsilon \times
$$$$
\sum_{n=1,3, \ldots}^{\infty} B_{n}\left\{p\left[p \delta_{n}\left(b_{13}+1\right) \sin \rho \delta_{n}-\left(b_{13}+2\right) \cos p \delta_{n}\right] \cos p \delta_{n} \eta-\right.
$$$$
\left.-\left(b_{13}+1\right) p^{2} \delta_{n} \cdot \eta \cos p \delta_{n} \cdot \sin p \delta_{n} \eta+0(\varepsilon)\right] \frac{d m_{n}}{d \xi} ;
$$$$
u_{\xi 0}=\left(b_{13}+1\right) p \times
$$$$
\sum_{n=1,3, \ldots}^{\infty}\left[C _ { n } \delta _ { n } \left\{\left[\sin p \delta_{n}+\frac{\cos p \delta_{n}}{p \delta_{n}\left(b_{13}+1\right)}\right] \sin p \delta_{n} \eta+\right.\right.
$$$$
\left.+\eta \cos p \delta_{n} \cdot \cos p \delta_{n} \eta+0(\varepsilon)\right\} m_{n}(\xi) \text {; }
$$$$
\sigma_{r 0}=G_{1} \sum_{n=1,3, \ldots}^{\infty} B_{n}\left\{\left[\left(b_{13}+1\right) p \delta_{n}\left(b_{13}-b_{11} p^{2} \delta_{n}\right) \sin p \delta_{n}+\right.\right.
$$$$
\left.+\left(b_{13}+b_{11} p^{2} \delta_{n}\right) \cos p \delta_{n}\right] \sin p \delta_{n} \eta+
$$$$
\left.+\left(b_{13}+1\right)\left(b_{13}-b_{11} p^{2} \delta_{n}\right) \cdot \eta \cos p \delta_{n} \cos p \delta_{n} \eta+0(\varepsilon)\right\} \frac{d m_{n}}{d \xi} ;
$$$$
\sigma_{\phi 0}=G_{1} \sum_{n=1,3 \ldots}^{\infty} C_{n}\left\{\left[\left(b_{13}+1\right) p \delta_{n}\left(b_{13}-b_{12} p^{2} \delta_{n}\right) \sin p \delta_{n}+\right.\right.
$$$$
\left.+\left(b_{13}+b_{12} p^{2} \delta_{n}\right)\right] \sin p \delta_{n} \eta+
$$$$
\left.+\left(b_{13}+1\right)\left(b_{13}-b_{12} p^{2} \delta_{n}\right) \cdot \eta \cos p \delta_{n} \cdot \cos p \delta_{n} \eta+0(\varepsilon)\right\} \frac{d m_{n}}{d \xi} ;
$$ 


$$
\begin{aligned}
& \sigma_{z 0}=G_{1} \sum_{n=1,3, \ldots}^{\infty} B_{n}\left\{\left[\left(b_{13}+1\right) p \delta_{n}\left(b_{33}-b_{12} p^{2} \delta_{n}\right) \sin p \delta_{n}+\right.\right. \\
& \left.+\left(b_{33}+b_{12} p^{2} \delta_{n}\right) \cos p \delta_{n}\right] \sin p \delta_{n} \eta+ \\
& \left.+\left(b_{13}+1\right)\left(b_{33}-b_{12} p^{2} \delta_{n}\right) \cdot \eta \cos p \delta_{n} \cdot \cos p \delta_{n} \eta+0(\varepsilon)\right\} \frac{d m_{n}}{d \xi} \\
& \tau_{r z 0}=\frac{2 G\left(b_{13}+1\right)}{\varepsilon} \times \\
& \sum_{n=1,3, \ldots}^{\infty} B_{n}\left[\delta_{n}^{3}\left(\sin p \delta_{n} \cdot \cos p \delta_{n} \eta-\eta \cos p \delta_{n} \cdot \sin p \delta_{n} \eta\right)+0(\varepsilon)\right] \times \\
& \times m_{n}(\xi)
\end{aligned}
$$

Expressions for $n=2,4,6, \ldots$ are obtained from the formulas (21), (22) by the replacement of $\cos x$ into $\sin x$ and of $\sin x$ into $-\cos x$ respectively.

$$
\begin{aligned}
& u_{\rho 0}=\varepsilon \sum_{n=1,3, \ldots}^{\infty} D_{n}\left\{\left[\left(b_{33}+\beta^{2}-\chi^{2}\right) \cos \beta \delta_{n} \eta \operatorname{ch} \chi \delta_{n} \eta+\right.\right. \\
& \left.+2 \chi \beta \sin \beta \delta_{n} \eta \cdot \operatorname{sh} \chi \delta_{n} \eta\right) \Delta_{1 n}- \\
& -\left[\left(b_{33}+\beta^{2}-\chi^{2}\right) \sin \beta \delta_{n} \eta \operatorname{sh} \delta_{n} \eta-\right. \\
& \left.\left.-2 \chi \beta \cos \beta \delta_{n} \eta \cdot \operatorname{ch} \chi \delta_{n} \eta\right) \Delta_{2 n}+0(\varepsilon)\right\} \frac{d m_{n}}{d \xi} \\
& u_{\xi 0}=\left(b_{13}+1\right) \times \\
& \sum_{n=1,3, \ldots}^{\infty} D_{n}\left[\left(\beta \sin \beta \delta_{n} \eta \operatorname{ch} \chi \delta_{n} \eta-\chi \cos \beta \delta_{n} \eta \cdot s h \chi \delta_{n} \eta\right) \Delta_{1 n}+\right. \\
& \left.+\left(\chi \sin \beta \delta_{n} \eta c h \chi \delta_{n} \eta+\beta \cos \beta \delta_{n} \eta \operatorname{sh} \chi \delta_{n} \eta\right) \Delta_{2 n}+0(\varepsilon)\right] \times \\
& \times m_{n}(\xi) ; \\
& \sigma_{r 0}=G_{1} \sum_{n=1,3, \ldots}^{\infty} D_{n}\left[b_{11} F_{n 1}^{\prime}(\eta)+b_{13} F_{n 2}(\eta)+0(\varepsilon)\right] \frac{d m_{n}}{d \xi} \\
& \sigma_{r z 0}=\frac{G}{\varepsilon} \sum_{n=1,3, \ldots}^{\infty} D_{n}\left[\delta_{n}^{2} F_{1 n}(\eta)+F_{n 2}^{\prime}(\eta)+0(\varepsilon)\right] m_{n}(\xi) ;(23) \\
& \sigma_{n=1,3, \ldots} \sum_{n=1,3, \ldots}^{\infty} D_{n}\left[b_{12} F_{n 1}^{\prime}(\eta)+b_{13} F_{n 2}(\eta)+0(\varepsilon)\right] \frac{d m_{n}}{d \xi} \\
& \sum_{n}^{\infty} D_{n}\left[b_{12} F_{n 1}^{\prime}(\eta)+b_{33} F_{n 2}(\eta)+0(\varepsilon)\right] \frac{d m_{n}}{d \xi}
\end{aligned}
$$

where

$$
\begin{aligned}
& F_{n 1}(\eta)=\left[\left(b_{33}+\beta^{2}-\chi^{2}\right) \cos \beta \delta_{n} \eta \operatorname{ch} \chi \delta_{n} \eta+\right. \\
& \left.+2 \chi \beta \sin \beta \delta_{n} \eta \operatorname{sh} \chi \delta_{n} \eta\right] \Delta_{1 n}- \\
& -\left[\left(b_{33}+\beta^{2}-\chi^{2}\right) \sin \beta \delta_{n} \eta \operatorname{sh} \chi \delta_{n} \eta-\right. \\
& \left.-2 \chi \beta \cos \beta \delta_{n} \eta \operatorname{ch} \chi \delta_{n} \eta\right] \Delta_{2 n}
\end{aligned}
$$

$$
\begin{aligned}
& F_{n 2}(\eta)=\left(\beta \sin \beta \delta_{n} \eta \operatorname{ch} \chi \delta_{n} \eta-\chi \cos \beta \delta_{n} \eta \operatorname{sh} \chi \delta_{n} \eta\right) \Delta_{1 n}+ \\
& +\left(\chi \sin \beta \delta_{n} \eta \operatorname{ch} \chi \delta_{n} \eta+\beta \cos \beta \delta_{n} \eta \operatorname{sh} \chi \delta_{n} \eta\right) \Delta_{2 n} \\
& \Delta_{1 n}=-\left[b_{33}+\left(b_{13}+2\right)\left(\beta^{2}-\chi^{2}\right)\right] \sin \beta \delta_{n} \operatorname{sh} \chi \delta_{n}+ \\
& +2 \chi \beta\left(b_{13}+2\right) \cos \beta \delta_{n} \operatorname{ch} \chi \delta_{n} ; \\
& \Delta_{2 n}=-\left[b_{33}+\left(b_{13}+2\right)\left(\beta^{2}-\chi^{2}\right)\right] \cos \beta \delta_{n} \operatorname{ch} \chi \delta_{n}- \\
& \quad 2 \chi \beta\left(b_{13}+2\right) \sin \beta \delta_{n} \operatorname{sh} \chi \delta_{n} ;
\end{aligned}
$$

Expressions for $n=2,4,6, \ldots$ are obtained from (23) by simply replacing $\operatorname{ch} \chi \leftrightarrow \operatorname{sh} \chi ; C_{n}, B_{n}, D_{n}$ are arbitrary constants.

We note that the solution (23) is characteristic only for anisotropic shells. It disappears completely in the transition to an isotropic shell $\left(G_{0}=1\right)$. With regard to the solutions (21) and (22) when $G_{0}=1$ they merge into one, and this solution coincides with the Saint Venant's solution for an isotropic plate.

In [32] a generalized condition of orthogonality of homogeneous solutions for the transverse isotropic hollow cylinder is proved, which allows to accurately satisfy the boundary conditions at the ends on special conditions of the shell edge bearing.

With the help of generalized orthogonality conditions, we consider the following problem: let the condition (3) satisfy on the side surface of the cylinder and the following boundary conditions be defined at the ends:

$$
\sigma_{z}=\lambda\left(1-c \eta^{2}\right), \quad u_{r}=0 \text { when } \xi= \pm l_{0},
$$

$2 l_{0}$ is the dimensionless height of the cylinder.

According to (21) $u_{r}, u_{z}, \sigma_{r}, \tau_{r z}$ can be written as

$$
\begin{array}{ll}
u_{r}=\sum_{n=1}^{\infty} C_{n} u_{n}(\eta) \frac{d m_{n}}{d z} ; \quad u_{z}=\sum_{n=1}^{\infty} C_{n} W_{n}(\eta) m_{n}(z) ; \\
\sigma_{z}=\sum_{n=1}^{\infty} C_{n} Q_{n}(\eta) \frac{d m_{n}}{d z} ; \quad \tau_{r z}=\sum_{n=1}^{\infty} C_{n} T_{n}(\eta) m_{n}(z) ;
\end{array}
$$

The summation in the series (24) is taken by the roots $\mu_{n}$ located in the upper half-plane $\left(\operatorname{Im} \mu_{n}>0\right)$. In view of the relations of a generalized orthogonality, the desired constants $C_{n}$ have the form:

$$
\begin{gathered}
C_{n}=-\lambda \Delta_{n}^{-1} c h \mu_{k} \ell_{0} \int_{-1}^{1}\left(1-c \eta^{2}\right) W_{n}(\eta) d \eta \\
\Delta_{n}=\int_{-1}^{1}\left[u_{n}(\eta) T_{n}(\eta)-Q_{n}(\eta) W_{n}(\eta)\right] d \eta .
\end{gathered}
$$

In general, the boundary value problem is reduced to solving systems of linear infinite algebraic equations using Lagrange variational principle. 


\section{Conclusion}

The main results obtained in the article, the following:

1) There are obtained simple asymptotic formulas allowing to find strain-deformed state of cylindrical shell with given precision;

2) There is distinguished a class of solution (23) which is characteristic only for anisotropic shells and totally disappear on passage to isotropic case;

3) It is shown that stress-strain state of a cylindrical shell is a sum of interior stress-strain state and countable set of boundary-layer solutions which is localized near the shell edge;

4) For $G_{0}=1$ boundary-layer solutions totally coincide with Saint-Venan solution for anisotropic plate.

By the same method there were investigated various problems some of which we consider [35], [36], [37], [38], [39], [40].

One of our authers (Mekhtiyev M.F.) devoted two monographs to the elaboration of asymptotic method of integrating the equations of anisotropic theory of elasticity for plates and shells of variable thickness [24], [25].

\section{References}

[1] Goldenveizer A. L. The theory of thin shells. M.: Nauka, 1976, $512 \mathrm{p}$.

[2] Goldenveizer A. L., Lidskiy V. B., Tovstik P. E. Free vibrations of thin elastic shells. M.: Nauka, 1979, 383 p.

[3] Novojilov V. V. Theory of elasticity. L.: Sudopromgiz, 1958, $370 \mathrm{p}$.

[4] Chernykh K. F. Linear shell theory. Part I, L.: LSU, 1962, 274 p.

[5] Chernykh K. F. Linear shell theory. Part II, L.: LSU, 1964, $395 \mathrm{p}$.

[6] Fridrichs K. O. Kirchoff's boundary conditions and the edge effect for elastic plates. // Proc. Simpos. Appl.. Mech., 1950.

[7] Fridrichs K. O. and Dressler R. F. A boundary layer theory for elastic plates.// Commun. Pure Appl. Math., 1961, vol. XIV, N1.

[8] Vorovich I. I. Some mathematical questions of the theory of plates and shells. // Proc.of the II-nd All Union Congress on theoretical and applied mechanics, 1964. Review reports, issue 3, M.: Nauka, 1966, pp. 116-136.

[9] Vorovich I. I., Kovalchuk V. Ye. On the basic properties of one system of homogeneous solutions. // Appl. math. and mech., 1967, vol. 31, issue 5, pp.861-869.

[10] Vorovich I. I. Some results and problems of asymptotic theory of plates and shells. // Proc. of I All Union school on theory and numerical methods of calculations of plates and shells. Tbilisi, 1975, pp. 51-150.

[11] Kolos A. V. On elaboration of classic theory of the bend of round plates. // Appl. math. and mech., 1964, vol.28, issue 3, pp.325-333.
[12] Kolos A. V. Methods of elaboration of classic theory of bend and stretching of plates. // Appl. math. and mech., 1965, vol.29, issue 4, pp.250-310.

[13] Agalovyan L. A. Application of the method of asymptotic integrating to the construction of the approximations of the theory of anisotropic shells. // Appl. math. and mech., 1966, vol.30, issue 2, pp. 388-393.

[14] Aksentyan O. K. On concentration of strains in thick plates. // Appl. math. and mech., 1966, vol.30, issue 5, pp. 963-970.

[15] Aksentyan O. K. Asymptotic analysis of the solutions to elasticity problems for plates at mixed boundary conditions. // Proc. of VIII All Union Conference on the theory of shells and plates. M., Nauka, 1973, pp.17-19.

[16] Malkina O. S. On the error in the definition of strain concentration on a free hole by the methods of plane elasticity theory. // Appl. math. and mech., 1968, vol.32, issue 6, pp.314-322.

[17] Malkina O. S. Strain-stress state of a thick plate on loading symmetric with respect to the median plane. Dissert. of the cand. of phys.-math. sci., Rostov-na-Donu, 1968, 171 p.

[18] Bazarenko N. A., Vorovich I. I. Asymptotic behaviour of the solution to an elasticity problem for a hollow cylinder of a finite length at a small thickness. // Appl.math. and mech., 1965, vol. 29, issue 6, pp.1035-1052.

[19] Bazarenko N. A., Vorovich I.I. Analysis of a threedimensional strain and deformed state of round cylindrical shells. Construction of elaborated applied theories. // Appl.math. and mech., 1969, vol. 33, issue 3, pp.495-510.

[20] Vilenskaya T. V., Vorovich I.I. Asymptotic behavior of the solution to an elasticity problem for a spherical shell of small thickness. // Appl.math. and mech., 1966, vol. 30, issue 2, pp. 278-295.

[21] Ustinov Yu. A. On some features of asymptotic method on its application to the study of the vibrations of thin nonhomogeneous elastic plates.// Proc. of I All Union school on theory and numerical methods of calculation of shells and plates. Tbilisi, 1975, pp.395-403.

[22] Ustinov Yu. A., Yudovich V. I. On completeness of a system of elementary solutions to a biharmonic equation in a semistripe. // Appl. math. and mech., 1973, vol.37, issue 4, pp.706714 .

[23] Ustinov Yu. A. Boundary value problems and the problem of limit passage from three-dimensional elasticity problems to two-dimensional ones for nonhomogeneous plates. Dissert. of the doctor of phys.-math. sci., M., 1977, 355 p.

[24] Mekhtiev M. F. Asymptotic analysis of some special elasticity problems for hollow solids, Baku, 2010, $316 \mathrm{p}$.

[25] Mekhtiev M. F. Method of homogeneous solutions in anisotropic shell theory. Baku, 2009, 334 p.

[26] Lidskiy V. B., Sadovnichiy V. A. Asymptotic formulas for the roots of one class of integer functions. Math.sbornik, 1968, N4, pp. 556-566.

[27] Mekhtiev M. F. Construction of dynamic elaborated theory for a hollow cylinder. // Intern. sbornik "Applied mathematics", Leningrad, LSU, 1988, pp. 207-211. 
[28] Akhmedov N. K., Mekhtiev M. F. Analysis of threedimensional elasticity problems for nonhomogeneous cut cone. // Russian Academy of Sciences, Appl. math. and mech., 1993, vol. 57, issue 5, pp. 113-119.

[29] Magsudov F. G., Mehdiyev M. F., Sadikhov P. M. The asymptotic theory for transversely isotropic hollow cylinder. // Proceedings of the $\mathrm{V}$ International Conference "Modern Problems of Continuum Mechanics", Rostov-on-Don, 2000, vol. 2, pp. 134-139.

[30] Mekhtiev M. F., Bergman R. M. Asymptotic analysis of dynamic problem of the theory of elasticity for transversely isotropic hollow cylinder. // Journal of Sound and Vibration, London, 2001, N 2, pp. 177-194.

[31] Mekhtiev M. F., Fomina N. I. Free vibrations of transversally isotropic hollow cylinders. Mechanics of Composite materials. New-York, 2002, vol.38, N1, pp. 55-67.

[32] Maksudov F. G., Mekhtiev M. F., Sadikov P. M. Construction of homogeneous solutions for a transversally isotropic hollow cylinder. Proceedings of IMM of Azerbaijan AS, 1999, vol. X, pp. 199-209.

[33] Lekhnitskiy S. G. Theory of elasticity of an anisotropic solid. M., Nauka, 1977, 415 p.

[34] Ambartsumyan S. A. General theory of anisotropic shells. M., Nauka, 1974, 448 p.
[35] Mekhtiyev M. F., Mardanov I. D., Amrahova A. R. Asymptotic analysis of bending problem for transversalisotropic plate of variable thickness. Trans. of NAS of Azerbaijan, series of phys.-tech. and math. sciences, Baku, 2002, N4, p. 223-236.

[36] Mekhtiyev M. F., Guseynov F. S. The construction of homogeneous solutions for transversally isotropic hollow sphere . Trans. of NAS of Azerbaijan, series of phys.-tech. and math. sciences, Baku, 2002, Vol. XXII, N1, p. 206-210.

[37] Mekhtiyev M. F. Asymptotic analysis of anisotropic elasticity theory problem for finite length hollow cylinder. Trans. of NAS of Azerbaijan, series of phys.-tech. and math. sciences, Baku, 2003, Vol. XXIII, N1, p. 219-228.

[38] Mekhtiyev M. F., Fomina N. I., Sardarli N. A. Torsion problem of transversally isotropic hollow cone of variable thickness. Proc. of IMM of NAS of Azerbaijan, XXIII, Baku, 2005, p. 199-206.

[39] Mekhtiyev M. F. Construction of homogeneous solutions of a non-axially-symmetric tension problem of elasticity theory for transversally isotropic plates of variable thickness. Trans. of NAS of Azerbaijan, series of phys.-tech. and math. sciences, Baku, 2006, XXVI, N1, p. 177-185.

[40] Mekhtiyev M. F. Non-axially-symmetric problem of elasticity theory for transversally isotropic hollow sphere. Trans. of NAS of Azerbaijan, series of phys.-tech. and math. sciences, Baku, 2007, XXVII, N1, p. 155-164. 